\title{
Relações de Gênero e a Atuação de Psicólogos na Oncologia: Subsídios para a Saúde Masculina
}

\author{
Alberto Mesaque Martins \\ Universidade Federal de Minas Gerais \\ Belo Horizonte, $M G$, Brasil \\ Andrea Pereira Gazzinelli \\ Instituto Mário Penna \\ Belo Horizonte, $M G$, Brasil \\ Virgínia Torres Schall \\ Centro de Pesquisa René Rachou - FIOCRUZ-MG \\ Belo Horizonte, $M G$, Brasil \\ Celina Maria Modena \\ Universidade Federal de Minas Gerais \\ Belo Horizonte, MG, Brasil
}

\begin{abstract}
RESUMO
O câncer vem sendo considerado um problema de saúde pública com maior índice de mortalidade entre a população masculina. Este estudo tem como objetivo identificar e analisar os relatos de psicólogos sobre a assistência voltada ao público masculino no âmbito da oncologia. Na perspectiva da Pesquisa Descritiva e Exploratória e dos Estudos de Gênero, foi elaborado um questionário semiestruturado respondido por 113 psicólogos de diferentes regiões do Brasil. Observou-se a incipiência de ações voltadas para o público masculino e um maior reconhecimento das necessidades específicas das mulheres. Os entrevistados apontaram para dificuldades de adesão dos homens às atividades dos serviços de psicologia e ao tratamento oncológico, revelando implicações dos aspectos socioculturais e institucionais. Os dados apontam para o processo de genderificação das instituições de saúde e a necessidade de inclusão do tema das masculinidades no âmbito do SUS.
\end{abstract}

Palavras-chave: Neoplasias. Gênero. Masculinidade. Atuação do psicólogo. Psicologia social.

\section{ABSTRACT}

Relationship Between Gender and the Action of Psychologists in Oncology: Subsidies to the Masculine Healthcare Cancer has been considered a problem of public health with the highest mortality ratings among the male population. This paper aims to identify and analyze the report of psychologists about the specialized attention towards the male public in the field of oncology. According to the Descriptive and Exploratory Research and the Gender Studies' perspective, a semi-structured questionnaire was elaborated and it was answered by 113 Brazilian psychologists. Through the answers, the lack of specific actions to the male public was observed in comparison to the recognition of the specific women's necessity. The interviewed ones pointed out the difficulties that men have to join the psychology services with the oncological treatment, exposing implications from the social-cultural and institutional aspects. The results points to the process of genderization of the health institutions and the necessity to include the theme of masculinity in the ambit of SUS.

Keywords: Neoplasm. Gender. Masculinity. Psychologist performance. Social psychology.

\section{RESUMEN}

\section{Relaciones de Género y la Práctica de los Psicólogos en la Oncología: Subsidios para la Salud Masculina}

El cáncer ha sido considerado un problema de salud pública con tasas de mortalidad más elevadas entre los hombres. Este estudio buscó identificar y analizar las opiniones de psicólogos sobre la asistencia dirigida al público masculino en la oncología. A la vista de la investigación descriptiva y exploratoria y de los estudios de género, fue criado un cuestionario semi-estructurado, respondido por 113 psicólogos del Brasil. Se observó una escasez de acciones dirigidas al público masculino y un mayor reconocimiento de las necesidades de las mujeres. Los entrevistados informaron las dificultades en la adhesión de los hombres a las actividades de los servicios de psicología y al tratamiento oncológico, revelando implicaciones de los aspectos socioculturales e institucionales. Los resultados relevan el proceso de genderificación de las instituciones de salud y la necesidad de inclusión de la cuestión de las masculinidades en él SUS.

Palabras clave: Neoplasias. Género. Masculinidad. Actuación del psicólogo. Psicología social. 


\section{INTRODUÇÃO}

Apesar do crescente investimento em estratégias para prevenção e diagnóstico precoce, o câncer, em suas múltiplas formas, ainda vem sendo considerado um importante problema de saúde pública, atingindo indivíduos de ambos os sexos, com diferentes idades e pertencentes a distintas classes sociais, despontando como a segunda causa de morte por doença em todo o mundo (NCIN, 2009; INCA, 2011). Segundo última estimativa do Instituto Nacional do Câncer (INCA), válida para os anos de 2012 e 2013, são esperados cerca de 518.510 novos casos da doença entre a população brasileira, sendo 260.640 entre as mulheres e 257.870 entre os homens (INCA, 2011).

Estudos apontam para o maior índice de mortalidade por câncer entre a população masculina, indicando uma maior vulnerabilidade dos homens a esta enfermidade. Sem desconsiderar a importância dos fatores biológicos, observa-se a necessidade de reflexão sobre as implicações de gênero na construção deste cenário (NCIN, 2009; White et al., 2010). O modelo hegemônico de masculinidade, construído, reforçado e idealizado em todo o processo de socialização contribui para a produção coletiva de representações acerca do "ser-homem", delineando assim, diferentes modos de exercício das masculinidades (Connel, 2005).

Tratando-se das sociedades latino-americanas, como a brasileira, marcadas pelas desigualdades de gênero e pela dominação masculina, aspectos como a coragem, a força física, a agressividade e a violência passam a ser reconhecidos como atributos masculinos, abrindo pouco espaço para manifestação de limitações e fragilidades como aquelas impostas pelas situações de adoecimento, favorecendo assim o distanciamento dos homens das ações de cuidado (Gomes, 2008).

Somam-se ainda as dificuldades recorrentes da maneira como os serviços de saúde são pensados e organizados, priorizando, na maior parte dos casos, o reconhecimento das necessidades das mulheres, crianças e idosos, contribuindo para o sentimento de não-pertencimento dos homens a estes espaços (Schraiber e Figueiredo, 2011). A dificuldade de incluir as demandas masculinas nas práticas de cuidado aponta para o processo de genderificação das organizações de saúde. Por se tratar de práticas sociais, concretizadas por sujeitos sócio-históricos e imersos em um contexto político, as questões de gênero invadem os serviços de saúde, orientando assim, os modos de pensar, sentir e agir tanto dos gestores e profissionais, como dos usuários e familiares que utilizam estes serviços (Lago e Muller, 2010; Machin et al., 2011).
Visando produzir mudanças neste cenário e disparar um processo de reflexão e construção de ações voltadas para a população masculina, foi instituída, em 2009, a Política Nacional de Atenção Integral à Saúde do Homem (PNAISH). Tendo em vista o crescimento de novos casos de câncer entre o público masculino, a PNAISH vem destacando a necessidade e viabilidade de formação dos profissionais de saúde para atendimento dos homens e incentivando o desenvolvimento de estratégias no âmbito da oncologia (Brasil, 2009).

As dificuldades de mobilização dos homens para ações de prevenção, diagnóstico precoce e controle do câncer vêm sendo apontadas como grandes desafios no planejamento das ações em oncologia (NCIN, 2009; White et al., 2010). O psicólogo vem sendo apontado como um importante ator que, aliado à equipe interdisciplinar e multiprofissional, pode favorecer a construção de espaços críticos-reflexivos que considerem e problematizem as implicações de gênero no processo de saúde/adoecimento/cuidado, favorecendo assim a expressão e o reconhecimento das demandas deste público (Lee e Owens, 2002; Martins et al., 2012).

É preciso levar em conta que o trabalho em saúde vai além da aplicação de técnicas padronizadas e do manejo de instrumentos tecnológicos. Antes, trata-se de um encontro entre sujeitos históricos, permeados por crenças, valores, sentidos e representações, construídos e compartilhados socialmente. Analisando as práticas dos profissionais de saúde Marques (2010) chama a atenção para o fato das mesmas encontraremse atravessadas pelas questões de gênero, contribuindo para a reprodução das desigualdades de gênero nas práticas de cuidado, revelando as implicações dos modos de produção da existência no campo da Saúde Coletiva.

Nessa perspectiva, este estudo tem como objetivo identificar e analisar os relatos de psicólogos sobre a assistência voltada ao público masculino no âmbito da oncologia.

\section{METODOLOGIA}

Trata-se de uma pesquisa descritiva e exploratória, que segundo Piovesan e Temporini (1995) possuem potencial de realizar uma análise prévia de uma realidade ou temática ainda pouco conhecida, levando em consideração as maneiras de sentir, pensar e agir do público-alvo e o contexto no qual ações de saúde serão desenvolvidas.

O referencial teórico que orientou a pesquisa foi a perspectiva de Gênero (Scott, 1995, Butler, 2010), sobretudo os estudos voltados para a compreensão da 
relação entre as masculinidades e os cuidados em saúde (Connel, 2005; Gomes, 2008). Conforme aponta Scott (1995) o conceito de gênero refere-se a "um elemento constitutivo de relações sociais baseadas nas diferenças percebidas entre os sexos e [...] uma forma primária de dar significado às relações de poder" (Scott, 1995, p. 86).

Somada às críticas contra a naturalização das diferenças sexuais, os estudos de gênero buscam no contexto sócio-histórico e político elementos que possibilitam compreender a maneira pela qual tais diferenças são transformadas em instrumentos de dominação e desigualdade social. Desse modo, gênero é tomado em sua dimensão discursiva, passando a constituir não apenas os corpos dos sujeitos, mas também seus discursos, permeando assim toda a vida social e orientando os modos, de pensar, sentir e agir (Butler, 2010).

Considerando-se o seu papel enquanto princípio ordenador e normatizador das relações e práticas sociais gênero contribui para a estruturação das percepções, emoções e ações dos profissionais que atuam no âmbito do cuidado em saúde (Machin et al., 2011). Imersos em um contexto sócio histórico, os profissionais de saúde (re)produzem em seu cotidiano de trabalho as desigualdades de gênero, produzindo mecanismos que potencializam as iniquidades no contexto da saúde. Nessa perspectiva, o conjunto de crenças e representações dos profissionais pode trazer implicações no planejamento e organização dos serviços de saúde, seja no nível da gestão, seja na forma como o cuidado em saúde será executado (Figueiredo e Schraiber, 2011).

Para coleta dos dados foi elaborado um questionário semi-estruturado, auto-administrado, segundo pressupostos de Breakwell et al. (2010). Trata-se de um instrumento composto por 41 questões, sendo 39 fechadas e 02 abertas, que foram divididas em 04 núcleos centrais, sendo o primeiro composto por 14 questões de caracterização do profissional respondente e o segundo por 04 itens sobre a instituição à qual encontra-se vinculado. O terceiro núcleo foi composto por 10 questões acerca das ações desenvolvidas no âmbito da oncologia e o quarto por 13 perguntas que buscavam identificar concepções acerca da assistência oferecida ao público masculino.

Responderam ao questionário, psicólogos que participaram do XI Congresso Brasileiro de PsicoOncologia e IV Encontro Internacional de Cuidados Paliativos, realizado na cidade do Rio de Janeiro RJ em Setembro de 2010. Optou-se pela aplicação dos questionários nesse evento tendo em vista o seu potencial de reunir psicólogos que atuam no âmbito da oncologia em diversas regiões do país.
Após autorização da coordenação do congresso, os questionários foram inseridos na pasta dos congressistas juntamente com um convite de participação, sendo recolhidos pela equipe de pesquisa ao final do evento. Compuseram o estudo aqueles psicólogos que consentiram em responder ao questionário. Os dados foram tabulados e analisados com auxílio do software SPSS, versão 12.0 .

O projeto de pesquisa foi aprovado pelo Comitê de Ética de Pesquisa do Centro de Pesquisas René Rachou - FIOCRUZ - pelo parecer $n^{\circ} 13 / 2010$, protocolo 03/2010 em conformidade com a Resolução 196/96 do Conselho Nacional de Saúde.

\section{RESULTADOS E DISCUSSÃO}

\section{Perfil dos entrevistados e das instituições}

Participaram deste estudo 113 psicólogos, sendo $103(91,1 \%)$ mulheres e 10 homens $(8,9 \%)$, com idade média entre 18 e 35 anos $(54,9 \%)$. O maior número de entrevistadas do sexo feminino reflete a maior presença das mulheres no âmbito da Psicologia e sua atuação significativa no campo da saúde coletiva, conforme já apontado por Bastos, Gondim e Borges-Andrade (2010) e por estudo realizado pelo Conselho Federal de Psicologia em 2010 (CFP, 2010). Entretanto, devese considerar que a distribuição do número de homens e mulheres entre as profissões regulamentadas no país também encontra-se permeado pelas questões de gênero.

Nesse sentido é preciso levar em conta o processo de divisão sexual do trabalho e do cuidado. Enquanto as profissões voltadas para a produção e administração dos meios que permitem a ampliação ou a destruição da vida humana têm sido associadas ao universo masculino, as atividades relacionadas ao cuidado passam a ser reconhecidas socialmente como ocupações femininas e, portanto, são muitas vezes desvalorizadas, podendo explicar o menor número de homens nessas ocupações (Lago e Muller, 2010). Estudos apontam que o expressivo número de mulheres que atuam nos serviços de saúde, bem como a maior freqüência de usuárias do sexo feminino, contribui para a feminilização destes espaços, contribuindo, muitas vezes, para o distanciamento dos homens e um menor número de ofertas assistenciais para este público (Gomes et al., 2011).

Tratando-se da formação acadêmico-científica dos profissionais entrevistados, observou-se uma significativa inserção dos psicólogos nos cursos de especialização $(73,5 \%)$ e uma menor participação nos cursos de mestrado $(15,9 \%)$ e doutorado $(6,2 \%)$. Deve-se considerar ainda, que $20 \%$ dos entrevistados 
referem-se a estudantes de graduação. Perfil semelhante foi apontado na última pesquisa encomendada Conselho Federal de Psicologia (CFP) ao Instituto de Pesquisa, Mercado e Opinião (WHO) no ano de 2001 (WHO, 2001).

Os dados parecem estar associados ao expoente crescimento dos cursos de Pós-Graduação Latu Senso nos últimos anos, bem como a maior busca de formação continuada pelos psicólogos (Seixas, CoelhoLima e Costa, 2010). Considerando-se a inserção dos entrevistados nos cursos de mestrado e doutorado, percebe-se a necessidade de maior investimento e participação dos profissionais no âmbito da pesquisa acadêmica voltada para a oncologia. Segundo Costa Junior (2001) é preciso investir na produção de conhecimento em Psico-Oncologia de modo que essa área não se restrinja à aplicação de técnicas, restritas ao contexto hospitalar.

Quanto à distribuição geográfica, observou-se maior concentração de respondentes na região Sudeste (59\%), seguidos das regiões Sul (13\%) e Nordeste (17\%). Houve uma menor representação das regiões Centro-Oeste $(8 \%)$ e Norte $(3 \%)$ do país. A distribuição geográfica dos psicólogos entrevistados traduz a realidade da distribuição destes profissionais no cenário brasileiro (CREPOP, 2010). Quanto à inserção profissional, 29\% dos psicólogos atuam em instituições particulares; $30 \%$ em filantrópicas; $24 \%$ em públicas e $17 \%$ em instituições que pertencem às três esferas. Resultados semelhantes são apontados nos levantamentos realizados pelo CFP (WHO, 2001).

Considerando-se os processos de trabalhos, $62 \%$ atuam em apenas uma instituição, 25,7\% em duas e $5,3 \%$ trabalham em três ou mais organizações, indicando uma característica dos trabalhadores da saúde que, em busca de complementação de renda, optam por exercer atividades laborais em mais de um contexto, podendo contribuir para sobrecarga de trabalho (Silva, 2010). A maior parte dos entrevistados $(35,5 \%)$ afirmou exercer suas atividades num período entre 20 e 35 horas semanais e apenas $18 \%$ relatou atuar em uma jornada semanal inferior a 20 horas. Chama a atenção o fato de $33 \%$ dos psicólogos participantes afirmar trabalhar em uma jornada superior a 40 horas semanais.

Quanto à experiência profissional no âmbito da oncologia, a maior parte dos participantes (41\%) atua neste contexto num período entre 01 e 05 anos, $15 \%$ entre 06 e 10 anos e $12,5 \%$ a mais de 10 anos. Apenas 15\% dos entrevistados afirmou trabalhar neste contexto específico num período inferior a 01 ano. Nesse sentido, participaram deste estudo, psicólogos que atuam no contexto da oncologia, em instituições públicas e privadas, nas diferentes regiões do país, com período significativo de experiência profissional.

\section{Masculinidades e o agir em saúde}

Considerando-se as atividades desenvolvidas pelos participantes, constatou-se o maior relato de ações voltadas para as mulheres com câncer, em detrimento daquelas disponibilizadas especificamente para o público masculino. Enquanto 29\% dos entrevistados alegaram realizar atividades específicas para as mulheres (grupos terapêuticos, oficinas psicoeducativas, dentre outros), apenas $7 \%$ afirmaram realizar algum tipo de ação voltada especificamente para o público masculino, indicando a incipiência de atividades que considerem as necessidades e demandas específicas dos homens nos serviços de oncologia.

A inexistência de ações para os homens foi justificada pelos participantes tendo em vista a "falta de tempo" (28\%); a "carência de profissionais" (28\%), a "ausência de espaço físico apropriado" (27\%) e ainda, a "necessidade de capacitação específica" (24\%). Sem negar a importância dos aspectos institucionais, sobretudo o delineamento de processos de trabalho que favoreçam a inclusão das necessidades masculinas nos serviços de saúde, observa-se que, embora importantes, esses elementos não inviabilizam a construção de ações voltadas para as mulheres, indicando uma possível valorização desse público no âmbito da saúde.

Diante da rotina de trabalho, muitas vezes sobrecarregada e com incipiência de recursos físicos, tecnológicos e humanos, gestores e profissionais de saúde optam pelo investimento nas necessidades específicas do público feminino, refletindo o seu reconhecimento social como sujeito universal dos cuidados e das políticas de saúde (Lago e Muller, 2010). Deve-se considerar que essa perspectiva encontra-se difundida nos discursos sexistas que permeiam a vida social, onde a mulher é considerada como o "sexo frágil" e o homem representado pela sua força física e invulnerabilidade. Desse modo, os profissionais de saúde acabam repetindo e reforçando os estereótipos de gênero, oferecendo um maior número de ações para os sujeitos que supostamente "mais precisam" e, de forma semelhante, um menor conjunto de atividades para aqueles que "não precisam", refletindo assim marcas dos modos de produção da existência e das desigualdades de gênero.

Soma-se ainda a maior familiaridade dos profissionais de saúde e o seu maior preparo acadêmico para atender às necessidades do público feminino. Nesse sentido, o silenciamento do tema das masculinidades nos espaços de formação universitária e de educação permanente contribui para uma prática profissional 
marcada por dificuldades no acolhimento e reconhecimento das demandas específicas da população masculina, que requer da equipe interdisciplinar um agir distinto daquele oferecido às mulheres (Schraiber et al., 2010). Tendo em vista o despreparo dos profissionais de saúde para atendimento dos homens é recorrente que suas demandas sejam abafadas e silenciadas produzindo a ideia de "resistência" e "baixa adaptação", sem levar em conta o fato de que, muitas vezes, as ações e serviços de saúde não são pensados considerando as singularidades e os modos de ser dessa população.

Resultados semelhantes foram encontrados em estudos recentes realizados no contexto brasileiro. Analisando as concepções de gênero e masculinidades de profissionais que atuam no âmbito da Atenção Básica, Machin et al. (2011) observaram que os mesmos reproduzem em seu cotidiano de trabalho estereótipos de gênero, representando as mulheres como "lócus de cuidado" e os homens como sujeitos resistentes, comprometendo assim o reconhecimento de suas necessidades e demandas específicas.

Em um estudo semelhante, Gomes et al. (2011) constataram que, apesar do aumento na procura dos homens pelos serviços de Atenção Primária, ainda é recorrente que os profissionais de saúde apontem para sua ausência destes espaços. Para os autores, esse fato indica o processo de invisibilidade dos homens nas instituições de saúde, bem como para a dificuldade de se representar o público masculino como sujeitos de cuidado de si e dos outros. Sem desconsiderar a menor frequência dos homens aos serviços de saúde é preciso reconhecer os processos de mudança, compreendê-los de modo que possam ser vislumbradas possibilidades de mudança e transformação.

No presente estudo, os participantes também apontaram para a dificuldade de adesão dos homens às atividades propostas pelos Serviços de Psicologia, bem como aquelas relacionadas ao tratamento oncológico, de modo geral. Nesse sentido, $69 \%$ dos entrevistados alegaram que os homens não aderem ou aderem com dificuldades às atividades dos serviços de Psicologia e $64 \%$ afirmaram que esse público ainda encontra grandes dificuldades para aderir e dar continuidade ao tratamento oncológico. Desde tenra idade os homens, ao contrário das mulheres, são incentivados a falarem pouco de si e a não expressar os seus sentimentos e emoções, sobretudo aquelas que possam tornar visíveis suas fragilidades e limitações. Desse modo, é recorrente que as ações em saúde, sobretudo aquelas voltadas para aspectos subjetivos sejam percebidas como uma ameaça que pode comprometer o reconhecimento de sua hombridade (Gomes, 2008).
Conforme destacam Martins et al. (2012) é preciso levar em conta que a condição imposta pelo adoecimento por câncer mostra-se incoerente com a posição ocupada pelos homens na sociedade, contribuindo para as dificuldades de adesão e adaptação à rotina de cuidados. Ainda nessa direção, é recorrente o sentimento entre os homens de que os serviços de saúde não respondem adequadamente às suas necessidades, contribuindo para a não adesão e identificação dos mesmos às atividades propostas e aos tratamentos ofertados (Gomes, 2008).

Considerando-se o seu processo de construção social, os homens requerem das equipes de saúde um modo singular de planejar e manejar as ações, diferentes daqueles propostos para crianças, mulheres e idosos (Gomes et al., 2011). Entretanto, novos modos de atuação demandam dos profissionais a busca por novos conhecimentos, a invenção de novas ações e a criação de perspectivas de trabalhos próprias. Entretanto, essas iniciativas esbarrem na sobrecarga de trabalho do psicólogo, que além dos atendimentos aos pacientes e familiares, pode incluir em seu cotidiano, trabalhos burocráticos e administrativos, bem como seleção, treinamento e desenvolvimento da equipe e, ainda, atendimento psicoterapêutico dos profissionais de saúde. Assim, é recorrente que a construção de ações voltadas para os homens nesses espaços seja percebida não como uma necessidade, mas sim, como uma nova demanda que se sobrepõe a um conjunto de tarefas de difícil realização.

Por outro lado, apesar da incipiência de atividades direcionadas para os homens nos serviços de oncologia onde atuam, $42 \%$ dos entrevistados afirmaram reconhecer em suas atividades profissionais cotidianas especificidades no atendimento desse público. As singularidades percebidas encontram-se ancoradas em pelo menos duas dimensões. A primeira refere-se às dificuldades do público masculino em adequar-se às atividades propostas pelos serviços de psicologia. Nesse sentido, os entrevistados apontaram para: "as características de personalidade masculina", "dificuldade para procurar suporte psicológico", "introspecção, insegurança, vergonha", dentre outros elementos que buscam no próprio sujeito explicações para o seu distanciamento.

Ainda nessa dimensão, foi enfatizado o papel da socialização na construção das especificidades do público masculino. Nesse sentido, alguns dos psicólogos apontaram para "a construção social da identidade do gênero masculino", a "educação", o "perfil de ser homem, macho na cultura", o "papel do masculino na nossa sociedade e funções a eles esperadas". Estas respostas parecem localizar a 
responsabilidade pelo distanciamento no próprio público masculino, desconsiderando o papel que os arranjos organizacionais e institucionais desempenham na construção e manutenção deste cenário (Schariber et al., 2010).

Resultados semelhantes vêm sendo evidenciados na literatura onde gestores e profissionais de saúde ainda encontram dificuldades de reconhecer o seu papel enquanto agentes de mobilização do público masculino para ações de cuidado, favorecendo assim a circulação de um discurso de culpabilização dos homens pelo próprio adoecimento e distanciamento dos serviços de saúde (Toneli, Souza e Muller, 2010; Machin et al. 2011). Deve-se atentar ainda para a recorrente utilização da categoria "cultura", muito relatada pelos profissionais entrevistados, enquanto perspectiva essencialista e explicativa das dificuldades de mobilização da população masculina.

Apesar do seu caráter constituinte e produtor de subjetividades, a cultura, assim como os aspectos biológicos, não podem ser tomados como únicos elementos explicativos para os modos de ser da população masculina. Portanto, é preciso romper com o viés determinista dos papéis de gênero, seja por elementos biológicos ou mesmos socioculturais, levando em conta que gênero refere-se a uma categoria complexa e em constante transformação (Connel, 2005).

A segunda dimensão, menos explicitada pelos entrevistados, diz respeito à dificuldade dos profissionais de saúde de lidarem com as demandas específicas do público masculino nos serviços de oncologia. Nesse sentido, alguns entrevistados afirmam: "creio que não é aberto espaço para eles (homens)", "(necessidade de) conhecer melhor as questões masculinas" e ainda "(necessidade de) conseguir atingir este público de forma que haja maior participação".

É preciso considerar a complexidade que envolve o afastamento dos homens dos serviços de saúde e as dificuldades dos profissionais em lidar com este público específico. Atribuir apenas aos homens ou ainda, somente aos profissionais de saúde a responsabilidade por este "divórcio" é cair numa armadilha simplista e incoerente. Faz-se necessário problematizar e compreender este distanciamento numa perspectiva sócio-histórica e contextualizada, na qual aspectos ligados à construção das masculinidades e à organização e estruturação dos serviços de saúde se articulam dando origem a uma complexa teia de significados.

A dificuldade de atendimento do público masculino foi referida por $27 \%$ dos entrevistados, indicando um sentimento de despreparo dos profissionais de saúde para lidar com as questões de gênero no âmbito da saúde, especialmente no que se refere às masculinidades. A inserção desta temática na formação em saúde, seja nos currículos de graduação ou de educação permanente, é apontada como um importante elemento com potencial de produzir sujeitos críticos e reflexivos, contribuindo para atendimentos nos quais o sujeito é considerado em sua totalidade, na perspectiva da integralidade (Martins et al., 2012).

Nessa direção, 12,5\% dos entrevistados afirmaram não se sentir preparados para trabalhar com o público masculino e $53 \%$ dos psicólogos afirmaram sentir necessidade de capacitação específica para subsidiar os trabalhos com os homens no âmbito da oncologia. Segundo Lee e Owens (2002) faz-se necessária a inclusão da temática das masculinidades no âmbito da Psicologia da Saúde, possibilitando a compreensão dos homens numa perspectiva relacional de gênero, situando-os em seu contexto político, social e cultural.

Observa-se também que a inclusão da temática das masculinidades no âmbito da oncologia ainda é uma questão incipiente, seja na literatura científica quanto no âmbito da pesquisa. Nesse sentido, 73\% dos respondentes afirmaram que, até o momento da pesquisa, não haviam participado de discussões acadêmico-científicas que envolvessem a temática das masculinidades. De forma semelhante, constatou-se a necessidade de maior investimento na divulgação da PNAISH entre os profissionais que atuam no âmbito da oncologia. No presente estudo, $77 \%$ dos entrevistados afirmaram desconhecer essa política pública e apenas $3,5 \%$ relataram ter participado de algum tipo de discussão acerca da sua implementação nos serviços de saúde onde atuam.

Por fim, mas não menos importante, chama a atenção nesse estudo o expressivo número de profissionais $(26 \%)$ que afirmaram não perceber a necessidade de considerar a categoria "gênero" nos serviços nos quais atuam, indicando para um possível abafamento das questões de gênero no contexto oncológico e a grande ênfase dada às premissas biomédicas no âmbito da assistência à saúde. Nessa perspectiva, a admissão no serviço de saúde, sobretudo durante a internação hospitalar, destitui o sujeito de suas singularidades, levando-o a assumir uma nova posição social: a de "paciente", remetendo a uma condição de assujeitamento e passividade frente a equipe profissional, levando em muitos casos, à despersonificação (Silva, 2009).

Ainda hoje é possível encontrar instituições nas quais os nomes dos sujeitos, símbolos de sua identidade e história pessoal, são substituídos por números e códigos referentes à patologia ou ao leito no qual 
estão institucionalizados (Silva, 2009). Tratando-se do publico masculino, percebe-se que este abafamento é potencializado tendo em vista o maior preparo e prática dos serviços de saúde em acolher as demandas femininas, em detrimento das necessidades masculinas (Machin et al., 2011).

\section{CONSIDERAÇÕES FINAIS}

Apesar dos avanços na luta pela efetividade dos princípios do SUS, muito há de se avançar no que tange à universalidade da assistência, à equidade do acesso, à integralidade do cuidado, sobretudo no que se refere à inclusão da temática de gênero nos serviços de saúde. Ainda hoje, propostas de transformação convivem com processos de trabalho orientados por perspectivas biomédicas que, muitas vezes, desconsideram o caráter histórico e político dos trabalhadores e usuários do SUS.

Os dados do presente estudo revelam o processo de genderificação das instituições de saúde que refletem os modos de produção da existência marcados pelas desigualdades de gênero. Nesse sentido, observou-se um incipiente número de ações voltadas para o público masculino com câncer e uma maior ênfase no atendimento das necessidades específicas das mulheres. Chama a atenção o grande número de profissionais que afirmam sentir-se despreparados para atender a população masculina, apontando para a necessidade de maiores investimentos em espaços de formação profissional que considerem as implicações de gênero nos processos de saúde/adoecimento/cuidado.

Os entrevistados apontam para as dificuldades de adesão do público masculino às atividades propostas pelos serviços de psicologia, bem como ao tratamento oncológico em geral, entretanto, desconhecem o papel da organização dos serviços de saúde na construção e manutenção deste distanciamento. Nesse sentido, os homens requerem das equipes um novo pensar, sentir e agir distinto daquele oferecido às mulheres, exigindo assim a (re)criação e a (re)invenção das práticas pelos profissionais.

Por outro lado, as equipes de saúde brasileiras ainda são marcadas pela sobrecarga de trabalho, incipiência de recursos físicos e tecnológicos, necessidades de ampliação do número de profissionais, revelando a necessidade de se levar em conta os aspectos institucionais na reflexão sobre a mobilização dos homens para os serviços de saúde. Desse modo, torna-se urgente a construção de arranjos organizacionais e o delineamento de processos de trabalho que possibilitem que os profissionais e gestores em saúde reflitam sobre a necessidade de inclusão dos homens nas ações no âmbito da oncologia e, coletivamente, construam alternativas que favoreçam o reconhecimento de suas demandas e necessidades.

Nesse contexto, a Psicologia e os psicólogos ocupam um importante papel, tendo em vista o seu potencial para problematizar as práticas de saúde vigentes e construir processos críticos-reflexivos sobre temas que ressaltem o caráter político, histórico e cultural dos diferentes sujeitos que compõem os serviços de saúde. Mais do que caminhos pré-definidos e bem estabelecidos, observa-se a necessidade de novos estudos e investigações que considerem a temática das masculinidades no âmbito da oncologia e que apontem para caminhos possíveis de atuação, não apenas do psicólogo mas de toda a equipe de saúde.

\section{REFERÊNCIAS}

Bastos, A. V. B., \& Gomide, P. I. C. (2010). O psicólogo brasileiro: sua atuação e formação profissional. In: Yamamoto, O. H., \& Costa A. L. F.. (Org.). Escritos sobre a profissão de psicólogo no Brasil (pp. 227-254). Natal: EDUFRN.

Brasil. Ministério da Saúde. (2009). Política nacional de atenção integral à Saúde do Homem: princípios e diretrizes. Brasília: Ministério da Saúde.

Breakwell, G. M., Fife-Schaw, C., Hammond, S., \& Smith, J. A. (2010). Métodos de pesquisa em psicologia. Porto Alegre: Artmed.

Butler, J. (2010). Problemas de gênero: feminismo e subversão da identidade. Rio de Janeiro: Civilização Brasileira.

CREPOP. Centro de Referencia Técnica em Psicologia e Políticas Públicas (2010). Pesquisas sobre atuação dos psicólogos. Acessado em abril, 2012, em: http://crepop.pol.org.br/

Connel, R. W. (2005). Masculinities. Los Angeles: Berkeley.

Conselho Federal de Psicologia - CFP. (2010). Atuação dos psicólogos nos serviços hospitalares do Sistema Único de Saúde - SUS: relatório descritivo preliminar de pesquisa. Brasília: Conselho Federal de Psicologia.

Costa Junior, A. L. (2001). O desenvolvimento da psico-oncologia: implicações para a pesquisa e intervenção profissional em saúde. Psicologia Ciência e Profissão, 21(2), 36-43.

Figueiredo, W., \& Schraiber, L. B. (2011). Concepções de gênero de homens usuários e profissionais de saúde de serviços de atenção primária e os possíveis impactos na saúde da população masculina, São Paulo, Brasil. Ciência \& Saúde Coletiva, 16 (supl. 1), 935-944.

Gomes, R. (2008). Sexualidade masculina, gênero e saúde. Coleção: Criança, Mulher e Saúde. Rio de Janeiro: FIOCRUZ.

Gomes, R., Moreira, M. C., Nascimento, E. F.; Rebello, L. E., Couto, M. T., \& Schraiber, L. B. (2011). Os homens não vêm! Interpretação dos profissionais de saúde sobre ausência e ou invisibilidade masculina nos serviços de atenção primária do Rio de Janeiro. Ciência e Saúde Coletiva, 16 (supl. 1), 983-992.

Instituto Nacional do Câncer - INCA (2011). Estimativa 2012: incidência de câncer no Brasil. Rio de Janeiro: Instituto Nacional do Câncer.

Lago, M. C. S., \& Muller, R. C. F. O sujeito universal do cuidado no SUS: gênero, corpo e cuidado com a saúde nas falas de profissionais e usuários do Hospital Universitário - Florianópolis, Santa Catarina. In Strey, M. N., Nogueira, C., \& Azambuja, M. R. (Orgs.). Gênero \& saúde: diálogos ibero-brasileiros (pp. 279-302). Porto Alegre: Edipucrs. 
Lee, C. \& Owens, R.G. (2002). Issues for a Psychology of Men's Health. Journal of Health Psychology, 7(3), 209-217.

Machin, R. et al. (2011). Concepções de gênero, masculinidade e cuidados em saúde: um estudo com profissionais de saúde da atenção primária. Interface: comunicação, saúde, educação, 14 (33), 257-270.

Marques, A. M. (2010). Gênero e Saúde: uma relação ainda oculta. In Strey, M. N., Nogueira, C., \& Azambuja, M. R. (Orgs.). Gênero \& saúde: diálogos ibero-brasileiros (pp. 35-58). Porto Alegre: Edipucrs.

Martins, A. M., Gazzinelli, A. P., Almeida, S. S. L., Schall, V. T., \& Modena, C. M. (2012). Concepções de psicólogos sobre adoecimento de homens com câncer. Psicologia: Teoria $e$ Prática.

National Cancer Intelligence Network - NCIN. (2009). The excess burden of câncer in men in the UK. Acessado em março, 2012, em: http://info.cancerresearchuk.org/prod_consump/groups/ cr_common/@nre/@sta/documents/generalcontent/crukmig 1000ast-2748.pdf

Piovesan, A., \& Temporini, E. R. (1995). Pesquisa exploratória: procedimento metodológico para o estudo de fatores humanos no campo da saúde pública. Revista de Saúde Pública, 29(4), 318-325.

Schraiber, L. B., \& Figueiredo, W. S. (2011). Integralidade em saúde e os homens na perspectiva relacional de gênero. In Gomes R. (Org.). Saúde do Homem em debate (pp. 19-38). Rio de Janeiro: Editora Fiocruz.

Schraiber, L. B. et al. (2010). Necessidades de saúde e masculinidades: atenção primária no cuidado aos homens. Cadernos de Saúde Pública, 26(5), 961-970.

Scott, J. (1995). Gênero: uma categoria útil de análise histórica. Educação \& Realidade, 20(2), 71-99.

Seixas, P. S., Coelho-Lima, F., \& Costa, A. L. F. (2010). Caracterização de dissertações/teses que versam sobre a profissão de psicólogo no Brasil. In Yamamoto, O. H., \& Costa A. L. F. (Orgs.). Escritos sobre a profissão de psicólogo no Brasil (pp. 59-98). Natal: EDUFRN, 2010.
Silva, L. C. (2009). O Cuidado na vivência do doente de câncer: uma compreensão fenomenológica. Maringá: UEM.

Silva, A. B. H. C. (2010). O estresse na prática profissional do psicólogo em UTI: uma revisão de literatura. Rev. SBPH, 13 (1), 33-51.

Toneli, M. J. F., Souza, M. G. C. \& Muller, R. C. F. (2010), Masculinidades e práticas de saúde: retratos da experiência de pesquisa em Florianópolis/SC. Physis, 20(3), 973-994.

White, A. K., Thomson, C. S., Forman, D., \& Meryn, S. (2010) Men's Health and the Excess Burden of Cancer in Men. European Urology Supplements, 9(3), 467-470.

Who - Instituto de Pesquisa Mercado e Opinião. (2001). Pesquisa feita junto aos associados do Conselho Federal de Psicologia. Relatório Final. CFP.

Autores:

Alberto Mesaque Martins - Psicólogo (Centro Universitário UNA de Belo Horizonte), Mestrando em Psicologia (UFMG), Professor Substituto no Departamento de Psicologia da Faculdade de Filosofia e Ciências Humanas (FAFICH) da Universidade Federal de Mias Gerais (UFMG), Belo Horizonte, MG, Brasil.

Andrea Pereira Gazzinelli - Psicóloga (PUC Minas), Especialista em PsicoOncologia (SBPO), Instituto Mário Penna-MG.

Virgínia Torres Schall - Psicóloga (PUC Minas), Doutorado em Educação (PUC-RJ), Pesquisadora Titular e Coordenadora do Laboratório de Educação em Saúde e Ambiente (LAESA) do Centro de Pesquisas René Rachou (CPqRR) da Fundação Oswaldo Cruz (FIOCRUZ-MG).

Celina Maria Modena - Psicóloga (UFMG), Pós-Doutorado em Saúde Coletiva (FIOCRUZ), Pesquisadora Colaboradora e Orientadora do Programa de Pós Graduação em Saúde Coletiva do Centro de Pesquisas René Rachou (CPqRR) da Fundação Oswaldo Cruz (FIOCRUZ-MG).

Endereço para correspondência:

Alberto Mesaque Martins

Departamento de Psicologia

Faculdade de Filosofia e Ciências Humanas (FAFICH)

Universidade Federal de Minas Gerais (UFMG)

Av. Antônio Carlos, 6627 - Pampulha

CEP: 31270-901 Belo Horizonte, MG, Brasil

Tel.: (31)3409-5027 ou (31)8873-5553

E-mail: albertomesaque@yahoo.com.br

Recebido em: 17.06.2013.

Aceito em: 27.09.2013. 